\title{
Las torres de defensa del litoral: Sant Vicent (Benicàssim) y Torrenostra (Torreblanca). Castellón, Spain. Estudio arquitectónico y constructivo
}

\author{
Alba Soler Estrela
}

Universitat Jaume I, Castellón, Spain, estrela@uji.es

\begin{abstract}
The towers of Sant Vicent and Torrenostra are part of the defensive network of watchtowers built along the Valencian coast in the 16th century. Despite the towers' importance and uniqueness they have barely been studied to date. These cases and their historical context are a major contribution to the history of architecture and construction, with their common features making a comparative study relevant. Their current condition should be understood as the result of several interventions over centuries, prompting a need for a proper interpretation of the definition of the original features. The study methodology is based on a thorough analysis of current condition and the documentation generated will support the specific studies, including identifying elements and building systems. The results should be interpreted within the historical and geographical context of the group of towers along the coast and provide data to typological repertoire.
\end{abstract}

Keywords: Watchtowers, Coastal towers, Historic architecture, Architectural Heritage.

\section{Introducción}

Las torres de Sant Vicent y de Torrenostra (situadas en Benicàssim $\mathrm{y}$ Torreblanca respectivamente), forman parte de la red defensiva de torres de vigilancia y defensa del litoral valenciano construidas entorno al siglo XVI.

Pese a su importancia y singularidad, no han sido suficientemente estudiadas hasta la fecha. Ambas torres pertenecen a lo que puede considerarse un subsistema defensivo del litoral entre Peñíscola y el rio Mijares (Boira, 2007). El tipo de elemento defensivo presenta variaciones según la geografía o relieve del litoral (acantilado medio o costas bajas) perteneciendo las torres estudiadas a este último caso.

Comparten una serie de características comunes que hace apropiado su estudio comparativo para la obtención de resultados. Su estado actual debe entenderse como resultado de diversas superposiciones a lo largo de los siglos, por lo que es necesaria su adecuada interpretación para la definición de los rasgos originales.

La investigación que se presenta es un avance de los trabajos actualmente en fase de realización (ver nota al final). La metodología de estudio se basa en la lectura constructiva de su estado actual, sin que hayamos podido disponer de catas ni ensayos. Se ha elaborado una primera documentación gráfica que sirve de soporte a un análisis arquitectónico y de identificación de elementos y sistemas constructivos.

\section{La torre de Sant Vicent}

Se encuentra junto al actual paseo marítimo, sobre la arena de la playa y en las proximidades del barranco Farchà. Protege l'Olla de 
Benicàssim y la misma villa. Se divisa la Torre Colomera de Oropesa y la sierra del Desierto de la Palmas.

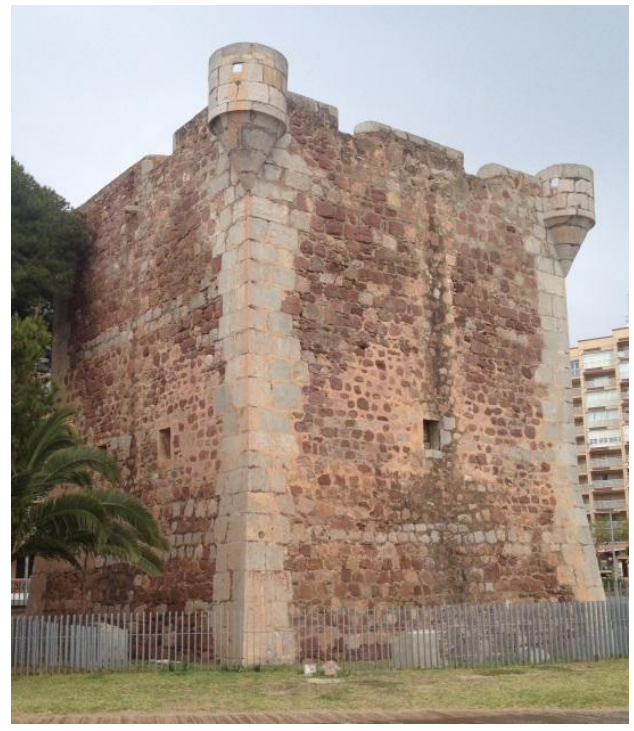

Fig. 1- Vista exterior de la torre de Sant Vicent.

Respecto a su datación, se conserva una importante fuente documental en relación con su construcción, como es el contrato original. Por ello se conoce que se comenzó en 1597, finalizándose en los primeros meses de 1599. Al parecer experimento reformas importantes en 1701 (Boira 2007).

La torre figura en el inventario de armamento y personal existente en las torres del distrito de Castellón, de 1728, hecho por mandato del Príncipe de Campoflorido y en él consta que estaba provista de: "un cañón de hierro de seis libras de calibre con su cureña de campaña, un atacador cuchara, sacatrapos, dos cavesales, dos cuñas de miras, dos espeques y doce balas para el cañón. Dos pasamuros, sinco mosquetes, sinco frascos, sinco horquillas, dos botavanes, siento $\mathrm{y}$ cuarenta bolas de mosquete, pólvora quinze libras, y cuerda mecha, cuatro varas y servían los soldados siguientes: Joseph Doménech, alcayde, Manuel Llopis y Pasqual Guiral, soldados de a cavallo, Joseph Prats y Lois Rovira, soldados de a pie" (Pérez-Olagüe en www.cult.gva.es).

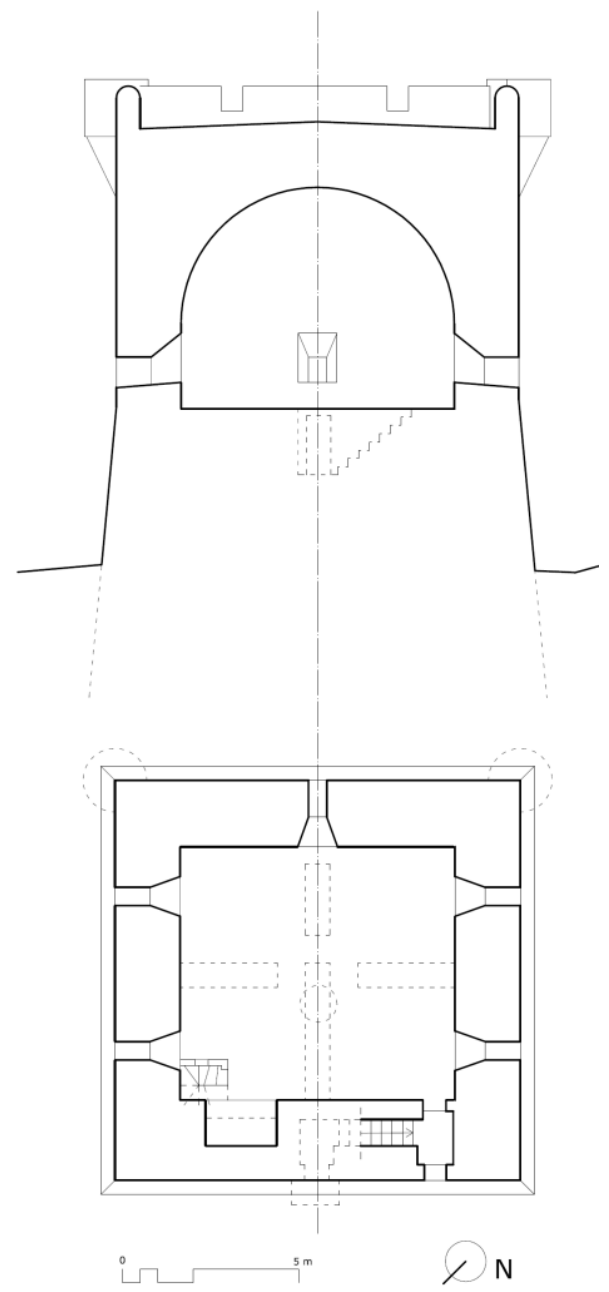

Fig. 2- Torre de Sant Vicent. Levantamiento inicial. Alba Soler, abril 2015.

\subsection{Descripción General}

Tiene planta cuadrada de unos $11.40 \mathrm{~m}$ exterior, con un interior de $7.75 \times 7.20 \mathrm{~m}$. Los muros tienen $1.90 \mathrm{~m}$ de espesor, excepto el del lado de la entrada de $2.27 \mathrm{~m}$ para permitir situar la escalera el acceso por el interior del muro. Estas dimensiones pueden relacionarse con la unidad de medida de la época. El espesor del muro sería de 1 cana de 8 palmos, o dos alnas de 4 (excepto el del acceso de 10 palmos), mientras que el interior sería de 32 palmos, obteniendo un total exterior de 50 palmos. 
Desde el exterior se observa como se eleva sobre una base ataluzada que da paso a los paramentos verticales coronados por dos matacanes cilíndricos (a modo de torrecillas) en la fachada recayente al mar y uno prismático sobre la puerta, en la fachada contraria.

Su única y gran sala interior se cubre por una bóveda de cañón de medio punto de $6.30 \mathrm{~m}$ de altura máxima.

Destaca la utilización de la cantería para la resolución de esquinas y elementos singulares combinada con la mampostería careada.

Está orientada en relación a la situación del mar (sur-este), de forma que el eje norte-sur corresponde con la diagonal.

\subsection{Elementos}

Base ataluzada o Escarpe:

La imponente base asciende sobre el nivel de arena actual, sin que podamos determinar su nivel de arranque. Analizando su interior, se puede observar el relleno de un opus cementicium de su total macizado. El paramento exterior de la base muestra los mismos materiales y técnicas que los muros superiores. Son de mampostería en la parte central del lienzo, pero reforzada en las esquinas por sillares aparejados con una altura que oscila entre los 40 y $60 \mathrm{~cm}$ con una longitud que llega hasta $100 \mathrm{~cm}$ (Fig. 1). Los sillares están colocados dejando adarajas y endejas para su traba con la mampostería. La inclinación de esta base, medida en la puerta de acceso produce un ensanchamiento de 1 medida por cada 10 de altura.

Muros:

Como se ha dicho, los muros mantienen la misma composición de mampostería reforzada con sillares en las esquinas, y que sirven además de referencia para conseguir la planeidad. Con toda probabilidad son muros tipo opus, formados por unas cortinas exteriores que contiene un relleno interior, similar al muro gótico de tres hojas. Algunos restos indican que todos los muros habrían estado revestidos. La hoja exterior de mampostería careada tendría un tratamiento de rejuntado de varios centímetros y un llagueado exterior con un mortero fino, que recibiría un fingido simulando juntas para mejorar el aspecto de la mampostería y relacionarla con las dimensiones de los sillares de las esquinas.

Bóveda:

Corresponde al tipo de bóveda más sencillo, de cañón de medio punto, apoyada en los muros norte-sur. En la clave tiene un gran espesor de casi $2 \mathrm{~m}$, lo que da muestras de una gran robustez relacionada con la seguridad. Se conservan las improntas en su intradós, que indican que para su construcción se emplearon cimbras de cañas (Fig. 3c). No se observa ninguna huella de vigas ni forjados intermedios, pero si un hueco cilíndrico en el centro de la bóveda, de dimensión libre próxima al metro. Se trataría de un hueco original al estar resuelto con cantería. Podría servir de acceso a la terraza mediante escalera de mano, para la subida de materiales y artillería y/o de iluminación y ventilación.

Vanos:

La sala dispone de cinco huecos distribuidos en sus alzados. Tanto el dintel como las jambas y el alfeizar están resueltos mediante sillares que se empotran en los muros de mampostería. La parte exterior es recta, produciéndose el abocinamiento para agrandarse en el interior. Los sillares presentan una textura que parece responder a un acabado picado con trinchante.

Puerta de acceso:

$\mathrm{Al}$ igual que el resto de vanos, está resuelta con sillares, con un dintel de dos hiladas con pequeña clave en segundo nivel (Fig. 3a). Es de pequeñas dimensiones 68 x $170 \mathrm{~cm}$ y está situada en el centro del lado opuesto al mar, dentro del nivel de la base ataluzada. Da paso a una estrecha escalera en recodo que transcurre por el interior del espesor del muro hasta el nivel de la sala interior.

Escalera:

Es de caracol, resuelta con bóveda tabicada. Tanto por su fábrica como por su situación en contradicción con chimenea, no se corresponde con la construcción original. Debió ejecutarse 
cuando la torre entro en obsolescencia, no obstante es una interesante muestra de construcción tradicional, emblemática solución de bóveda tabicada en espiral o caracol (Fig. 3b).

\section{Chimenea:}

Resuelta mediante cantería está formada por un arco muy rebajado, que es el único puesto que el resto de dinteles son siempre rectos.

Para ubicarla se produce vaciado del muro en $2 / 3$ de su espesor. Se ha perdido el remate superior original en la azotea.
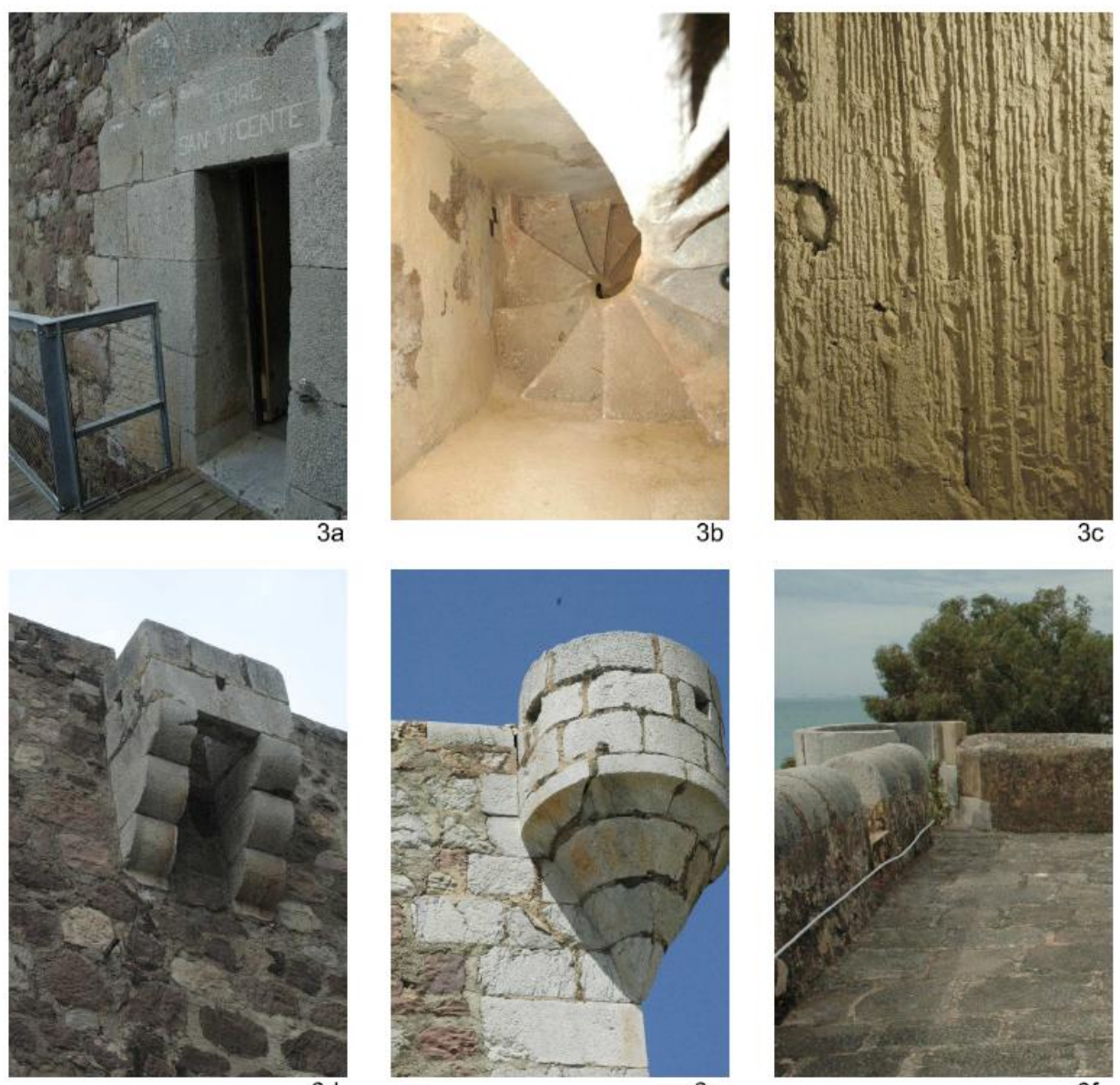

$3 e$

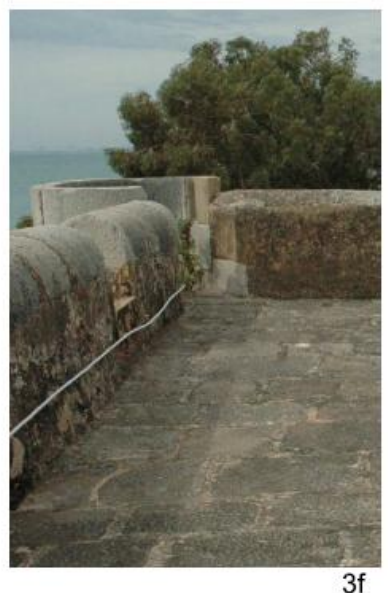

La mayor parte de la sala está sin pavimento, mostrando la capa inferior formado por mampuestos irregulares, suponemos que era el relleno de la base de la torre. En algunas zonas se conserva un pavimento de baldosas de barro. En una esquina de la chimenea se observa una losa de piedra que creemos puede ser el pavimento original de la torre, en consonancia con el contexto de sillares.

Fig. 3- Imágenes de la Torre de Sant Vicent.

Parapetos y remates superiores:

Los antepechos de la terraza están rematados con sillares de sección superior semicilíndrica 
En los dos vértices superiores de la fachada que mira al mar aparecen sendas torrecillas circulares voladas (Fig. 3e). Están formadas por sillares que crean una sucesión de ménsulas en forma de cono que se traba con los muros de mampostería. Sobre la puerta de acceso tiene un matacán rectangular (Fig. 3d).

\section{Cubierta:}

Es del tipo azotea (Fig. 3f), que se descompone en tres faldones mediante tres limatesas que vierten sus aguas a través de tres gárgolas gracias a la situación de las correspondientes limahoyas. Su acabado superior es de losas de piedra, aunque actualmente hay una zona con pavimento cerámico.

\subsection{Valoración}

El análisis de la torre muestra su importancia, sus grandes dimensiones y robustez, así como la existencia de un proyecto unitario y la calidad en su ejecución, destacando la técnica de cantería para las esquinas y puntos singulares.

La torre conserva un alto grado de autenticidad, salvo ciertas intervenciones claramente identificables debidas a sus últimos usos como cuartel del Cuerpo de Carabineros y luego de la Guardia Civil, entre las que destacan los muros que dividen actualmente la gran sala en 4 partes.

\section{La torre de Torrenostra}

Se ubica en el límite norte de un humedal difícil de atravesar, que hoy es el Parque Natural del Prat de Cabanes. Servía de protección a la cercana población de Torreblanca. Respecto a su datación, la primera referencia documental conocida son las Ordenanzas de 1673 (Boira 2007).

\subsection{Descripción}

Tiene planta cuadrada de unos $9 \mathrm{~m}$ en el exterior, con un interior de $4.45 \mathrm{~m}$. Los muros tienen $2.27 \mathrm{~m}$ de espesor. Estas dimensiones pueden relacionarse con la unidad de medida de la época. El espesor del muro es de 10 palmos, mientras que el interior es de 20, obteniendo un total exterior de 40 palmos. La altura total entre el final de la base inclinada y la cornisa es de
$9 \mathrm{~m}$, por lo que esta fachada es un cuadrado de 40 palmos.

Dispone de una sala en cada uno de sus tres niveles cubierta por bóveda de cañón de medio punto. La terraza superior, está rematada por cuatro torrecillas cilíndricas y un matacán rectangular sobre la puerta de entrada (actualmente en medianera cegada y por debajo del nivel del paseo). En el año 2006 fue restaurada, realizándose la reconstrucción de partes desaparecidas, especialmente a nivel de la terraza.

Al igual que la de Sant Vicent, su orientación es debida a la del mar que se encuentra al sur-este.

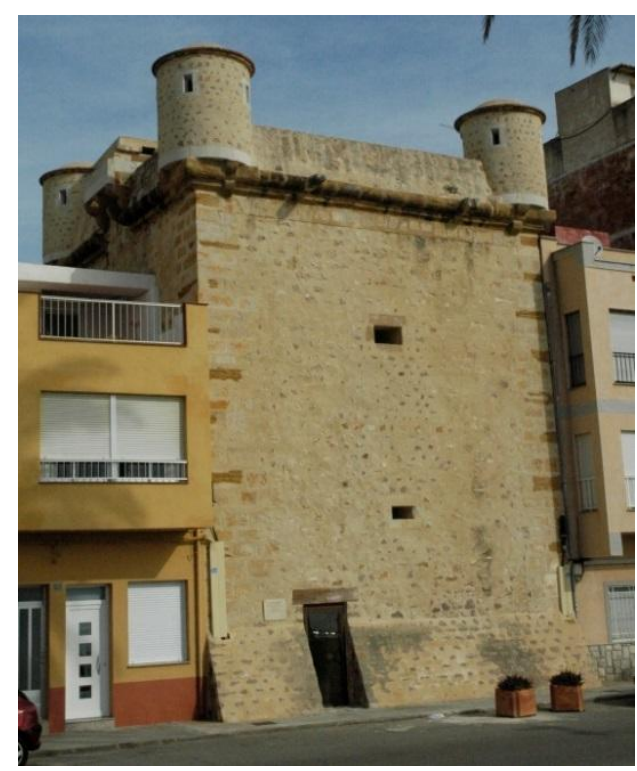

Fig. 4- Vista exterior de la torre de Torrenostra.

\subsection{Elementos}

Base y muros:

Aunque los niveles de las aceras actuales están muy por encima de los antiguos, se observa en parte una base ataluzada. Se estrecha a medida que asciende, con una inclinación muy acusada, que no podemos asegurar si corresponde con la original. Sobre esta base arrancan los muros, cuyos paramentos verticales combinan la mampostería reforzada con sillares en las esquinas (Fig. 3a). Se remata superiormente con una cornisa con moldura de piedra. 


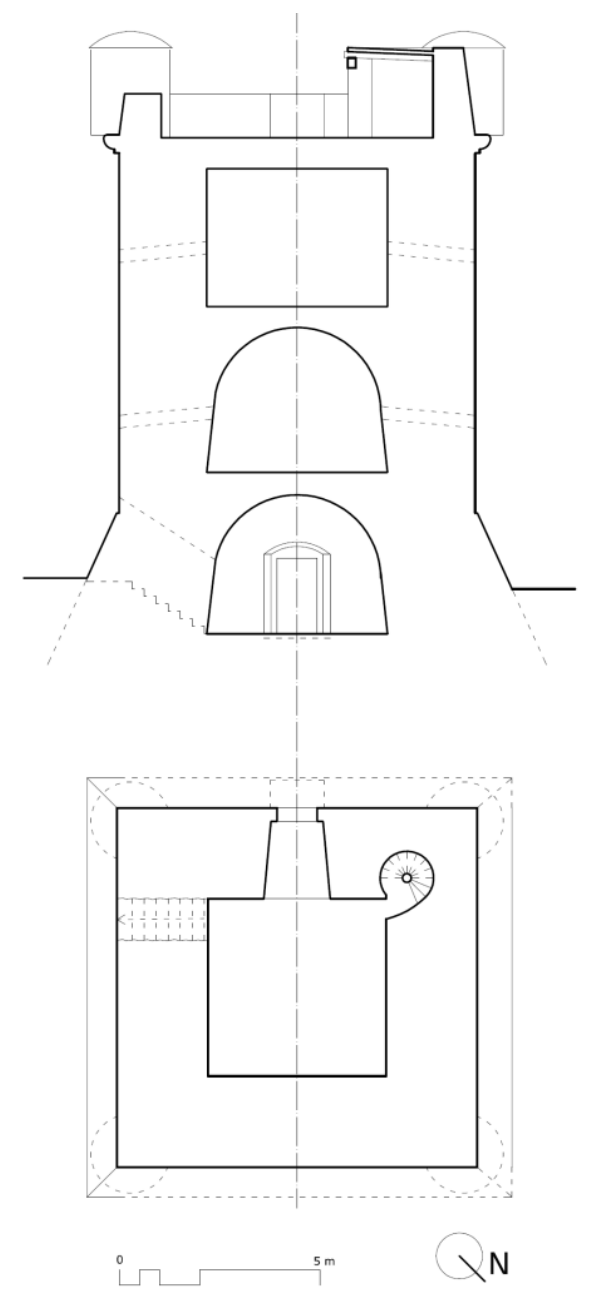

Fig. 5- Torre de Torrenostra. Levantamiento inicial. Alba Soler, abril 2015.

Bóvedas:

Las salas de los tres niveles están cubiertas por tres bóvedas del mismo tipo, de cañón de medio punto. Las del primer y segundo nivel se desarrollan en un sentido, mientras que la del tercero lo hace en el perpendicular. Aunque las bóvedas han sido restauradas pueden detectarse las huellas de las cimbras originales de tablas de madera de unos $26 \mathrm{~cm}$.

Chimeneas:

En las salas superiores, unos vaciados en los muros de mampostería sirven para situar el hogar. Se desconoce si son de la época inicial. Las campanas son de reciente construcción.

Vanos:

Existen una serie de pequeñas aberturas a modo de aspillera resueltas con sillares y mampuestos. Destaca una ventana adintelada actualmente cegada por la medianera, situada en la segunda planta, sobre la puerta de acceso original (Fig. 3c).

Puerta de entrada:

Tiene jambas de sillares, mientras que el dintel es una bóveda rebajada con improntas de cimbra de madera. El hueco de paso es de 1 x $1.90 \mathrm{~m}$. Daba acceso al nivel inferior, actualmente está cegada por la medianera y por debajo del nivel de aceras (Fig. 6b).

\section{Escalera:}

Es de caracol con nabo central, con un total de 6 palmos de diámetro interior. Discurre embebida en los muros, situada en una esquina. Es un elemente destacable, al ser de cantería con una buena ejecución de sus peldaños de piedra tallada (Fig. 6d).

\section{Pavimentos:}

Es de baldosa de barro de colocación reciente. No se han podido localizar restos del pavimento original.

Parapetos y remates superiores:

Esta zona es la más afectada por la intervención en el 2006, en la que construyeron cuatro torrecillas cilíndricas en las esquinas (Fig. 3f) y un matacán rectangular sobre la puerta de entrada.

\section{Azotea:}

La cubierta es una azotea plana con pavimento de piedra. Esta terraza tiene una parte cubierta con forjado de viguetas de madera de factura reciente, que parece haber tenido en cuenta alguna preexistencia.

\subsection{Valoración}

Desafortunadamente hoy se encuentra entre medianeras por lo que solo puede observarse la fachada que mira al mar y la posterior. Su estado 
actual se debe a la intervención llevada a cabo en 2006 con la reconstrucción de elementos, especialmente la parte superior. Las fotos anteriores muestran que estaba bastante degradada y afectada por construcciones impropias, como vanos y balcones. Aunque es necesario una lectura crítica respecto a su autenticidad, conserva elementos de gran interés.

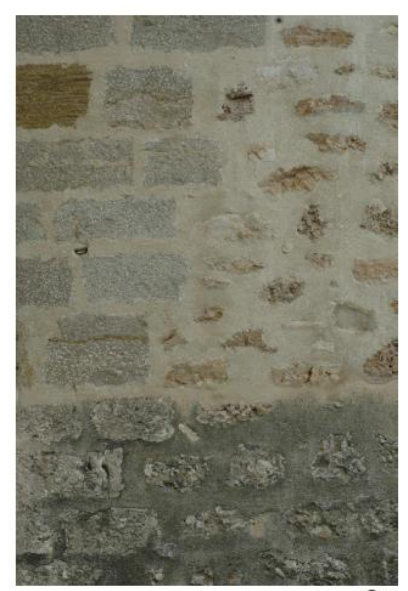

$6 a$

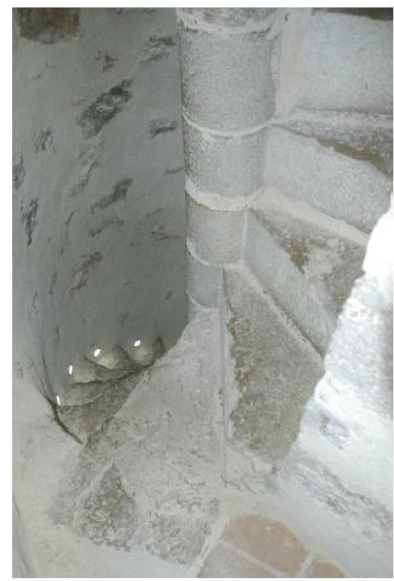

$6 d$

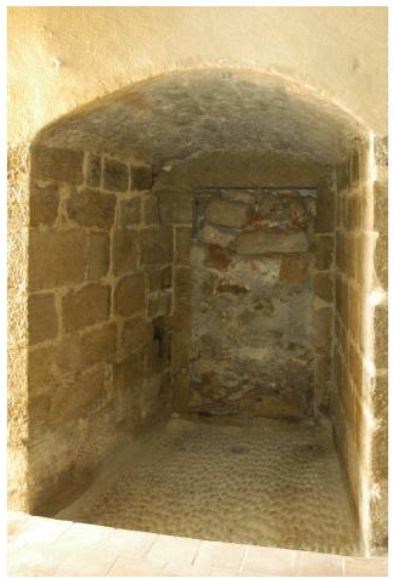

$6 b$

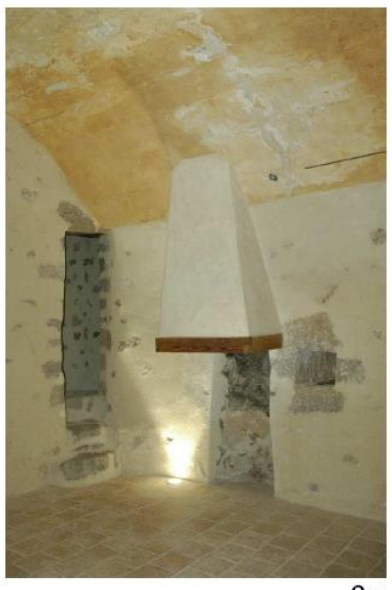

$6 e$

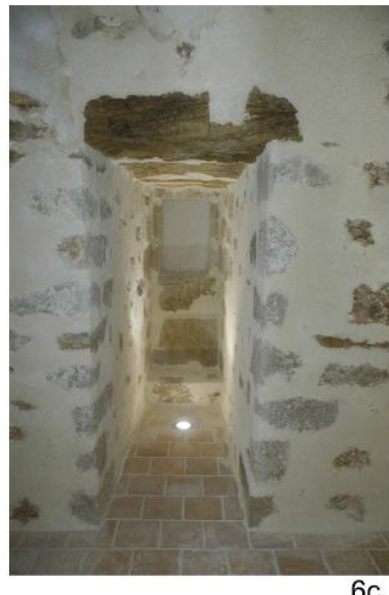

$6 c$

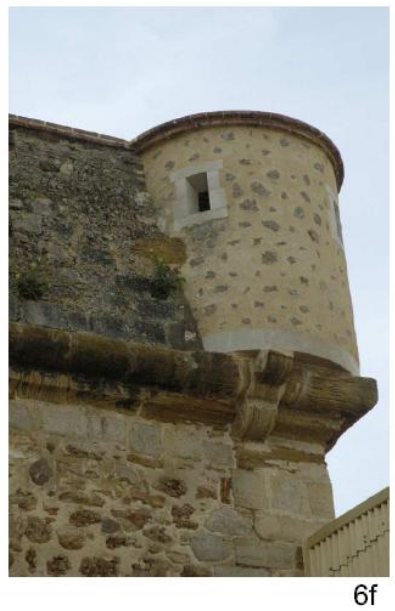

Fig. 6- Imágenes de la Torre de Torrenostra.

\section{Conclusiones}

Las torres estudiadas pertenecen al tipo funcional de torre de defensa, situadas en puertos, costas bajas o playas.

Respecto a las fecha de construcción, según los datos que hemos podido manejar, la torre de Torrenostra pudo ser construida tan solo unas décadas posteriores a la de Sant Vicent, en cualquier caso antes de 1673.
En un análisis inicial se aprecian similitudes, como su planta cuadrada, aunque varían sus dimensiones y la proporción de espesor de muro.

Frente a la abstracción tipológica, los modelos construidos responden a un repertorio variado en que cada torre adquiere su singularidad.

Una diferencia notable es que la de Sant Vicent tiene un solo nivel sobre una gran base macizada, en cambio la de Torrenostra tiene 
salas en tres niveles abovedados. Otra diferencia es la situación y forma de resolver el acceso, y también la escalera de caracol de sillería alojada en los muros de Torreblanca, que no tiene correspondencia en Benicàssim.

Respecto a las técnicas y sistemas constructivos, en Sant Vicent se utiliza una cantería de gran calidad en todos los puntos singulares, mientras que en Torrenostra su uso es más limitado, a excepción de la citada escalera de caracol. Otras diferencias son estilísticas, como la aparición de la cornisa o bordón superior de Torrenostra.

La presente aportación, junto con otros estudios de torres del mismo tipo, permitirá realizar generalizaciones y estudios comparativos dentro de esta línea abierta de investigación.

\section{Agradecimientos}

A Juan Domènech Mansilla por su colaboración en la toma de datos y medidas de Torrenostra.

A Rafael Soler Verdú por su asesoramiento y por su colaboración en la toma de datos en la torre de Sant Vicent.

\section{Notas}

La presente aportación se ha realizado dentro del Proyecto I+D del Programa Estatal de Investigación Científica y Técnica de Excelencia, Subprograma Estatal de Generación de Conocimiento, financiado por el Ministerio de Economía y Competividad, que lleva por título "Torres de vigía y defensa del litoral valenciano. Generación de metadatos y modelos 3D para su interpretación y efectiva puesta en valor", referencia HAR2013-41859-P.

\section{Referencias}

Arcienaga García, L. (1999) «Defensas a la antigua y a la moderna en el Reino de Valencia durante el siglo XVI» en Espacio, tiempo y forma. Serie VII, Historia del Arte, pp.61-94.

Boira Maiques, J. V. (2007) Las torres del litoral valenciano. Generalitat Valenciana

Cooper, E. (1994) The sentinels of Aragon. Old Coastal defense towers of Catalonia and Valencia. London Guildhall.

Serra Rodríguez, J.J. (2000) Fortificacions rurals a l'illa d'Eivissa. Les torres de refugi predials. Editorial Mediterranea.

Vila Bodoque, R. (2013) Torres de vigía y defensa del litoral valenciano (I): de la Torre de Sol de Riu a la Torrenostra. Proyecto Fin de grado. Tutor: Pablo Rodriguez. Inédito. Universidad Politécnica de Valencia.

Servicio de Patrimonio Cultural. Conselleria d'Educació, Cultura i Esport. Generalitat Valenciana. http://www.cult.gva.es/dgpa/arquitectonico_c.html (consulta abril 2015) 